\title{
Sum of weighted records in set partitions
}

\author{
Walaa Asakly \\ Department of Computer Science, University of Haifa, 3498838 Haifa, Israel \\ walaa_asakly@hotmail.com
}

\begin{abstract}
The purpose of this paper is to find an explicit formula and asymptotic estimate for the total number of sum of weighted records over set partitions of $[n]$ in terms of Bell numbers. For that we study the generating function for the number of set partitions of $[n]$ according to the statistic sum of weighted records.
\end{abstract}

Keywords: Records, Sum of weighted records, Set partitions, Generating functions, Bell numbers and Asymptotic estimate.

\section{Introduction}

Let $\sigma_{i}$ be an element in the permutation $\sigma=\sigma_{1} \sigma_{2} \cdots \sigma_{\ell}$, we say that $\sigma_{i}$ is a record of position $i$ if $\sigma_{i}>\sigma_{j}$ for all $j=1,2, \cdots, i-1$. The study of records in permutations interested Rényi [7]. More recently another statistic which depends on records have been studied by Kortchemski [5] who defined the statistic srec, where $\operatorname{srec}(\sigma)$ defined as the sum of the positions of all records of $\sigma$. For example, permutation $\pi=12534$ has 3 records, $1,2,5$ and $\operatorname{srec}(\pi)=1+2+3=6$. For relevant papers about records you can see for example 2 and 3 . In this Paper we want to focus on partitions of a set. Recall that a partition $\Pi$ of set $[n]$ of size $k$ (a partition of $[n]$ with exactly $k$ blocks) is a collection $\left\{B_{1}, B_{2}, \ldots, B_{k}\right\}$, where $\emptyset \neq B_{i} \subseteq[n]$ for all $i$ and $B_{i} \cap B_{j}=\emptyset$ for $i \neq j$, such that $\bigcup_{i=1}^{k} B_{i}=[n]$. The elements $B_{i}$ are called blocks, and we use the assumption that $B_{1}, B_{2}, \cdots, B_{k}$ are listed in increasing order of their minimal elements, that is, $\min B_{1}<\min B_{2}<\cdots<\min B_{k}$. The set of all partitions of $[n]$ with exactly $k$ blocks is denoted by $P_{n, k}$ and $\left|P_{n, k}\right|=S_{n, k}$, which is known as the Stirling numbers of the second kind [8]. And the set of all partitions of $[n]$ is denoted by $P_{n}$ and $\left|P_{n}\right|=\sum_{k=1}^{n} S_{n, k}=B_{n}$, which is the $n$-th Bell number $\left[8\right.$. Any partition $\Pi$ can be written as $\pi_{1} \pi_{2} \cdots \pi_{n}$, where $i \in B_{\pi_{i}}$ for all $i$, and this form is called the canonical sequential form. For example $\Pi=\{\{12\},\{3\},\{4\}\}$ is a partition of [4], the canonical sequential form is $\pi=1123$. For more details about set partitions we suggest Mansour's book [6. The important results about records, obtained by Knopfmacher, Mansour and Wagner [4] which state the asymptotic mean value and variance for the number, and for the sum of positions, of record in all partitions of $[n]$ are central to my study. In this paper, we define a new statistic swrec, where $\operatorname{swrec}(\pi)$ is the sum of the position of a record in $\pi$ multiplied by the value of the record over all the records in $P_{n}$. We will study this statistic from the point of view of canonical sequential form. For instance, if $\pi=121132$ the $\operatorname{swrec}(\pi)=1 \cdot 1+2 \cdot 2+3 \cdot 5=20$. 


\section{Main Results}

\subsection{The ordinary generating function for the number of set partitions ac- cording to the statistic swrec}

Let $P_{k}(x, q)$ be the generating function for the number of partitions of $[n]$ with exactly $k$ blocks according to the statistic swrec, that is

$$
P_{k}(x, q)=\sum_{n \geq k} \sum_{\pi \in P_{n, k}} x^{n} q^{\operatorname{swrec}(\pi)} .
$$

Theorem 1 The generating function for the number of partitions of $[n]$ with exactly $k$ blocks according to the statistic swrec is given by

$$
P_{k}(x, q)=\prod_{i=1}^{k} \frac{x q^{i} q^{(k+1-i)(k-i)}}{1-i x \prod_{j=i+1}^{k} q^{j}} .
$$

Proof As we know, a set partition of $[n]$ with exactly $k$ blocks can be presented as canonical sequential form:

$$
\pi=1 \pi^{(1)} 2 \pi^{(2)} \cdots k \pi^{(k)}
$$

for some $k$, where $\pi^{(j)}$ denotes an arbitrary word over an alphabet $[j]$ including the empty word. Thus, the contribution of $\pi=1 \pi^{(1)} 2 \pi^{(2)} \cdots k$ to the generating function $P_{k}(x, q)$ is $x q^{k} P_{k-1}\left(x q^{k}, q\right)$ and the contribution of $\pi^{(k)}$ to the generating function $P_{k}(x, q)$ is $\frac{1}{1-k x}$. Therefore, the corresponding generating function satisfies

$$
P_{k}(x, q)=\frac{x q^{k}}{1-k x} P_{k-1}\left(x q^{k}, q\right)
$$

By using induction on $k$ together with the initial condition $P_{1}(x, q)=\frac{x q}{1-x}$ we obtain the required result.

\subsection{Exact and asymptotic expression for $\sum_{\pi \in P_{n}} \operatorname{swrec}(\pi)$}

In this section, we aim to prove that the total number of the swrec over all partitions of $[n]$ is

$$
\frac{3}{4}\left(B_{n+3}-B_{n+2}\right)-\left(n+\frac{7}{4}\right) B_{n+1}-\frac{1}{2}(n+1) B_{n}
$$

And we want to show that asymptotically the total number of the swrec over all partitions of $[n]$ is

$$
B_{n} \frac{n^{3}}{r^{3}}\left(1+\frac{r}{n}\right)\left(1+O\left(\frac{\log n}{n}\right)\right),
$$


where $r$ is the positive root of $r e^{r}=n+1$.

For that we need to perform the following steps:

- Firstly, we find the partial derivative of $P_{k}(x, q)$ with respect to $q$ and substitute $q=1$, that is $\left.\frac{d}{d q} P_{k}(x, q)\right|_{q=1}$.

- Secondly, we pass from $\left.\frac{d}{d q} P_{k}(x, q)\right|_{q=1}$ to $\left.\frac{d}{d q} \widetilde{P}_{k}(x, u, q)\right|_{u=q=1}$, where $\widetilde{P}_{k}(x, u, q)$ is the exponential generating function for the number of partitions of $[n]$ with exactly $k$ blocks according to the statistic swrec.

- Finally, we derive the total number of swrec over all partitions of $[n]$, and the asymptotic estimate for the total number of swrec over all partitions of $[n]$.

Lemma 2 For all $k \geq 1$,

$$
\left.\frac{d}{d q} P_{k}(x, q)\right|_{q=1}=\frac{x^{k}\left(\left(\begin{array}{c}
k+1 \\
2
\end{array}\right)+2\left(\begin{array}{c}
k+1 \\
3
\end{array}\right)\right)}{(1-x) \ldots(1-k x)}+\frac{x^{k+1}}{(1-x) \ldots(1-k x)} \sum_{i=1}^{k} \frac{i(i+1+k)(k-i)}{2(1-i x)} .
$$

Proof By differentiating (1) with respect to $q$, we obtain

$$
\left.\frac{d}{d q} P_{k}(x, q)\right|_{q=1}=P_{k}(x, 1) \sum_{i=1}^{k} \lim _{q \rightarrow 1}\left(\frac{\frac{d}{d q} L_{i}(q)}{L_{i}(q)}\right),
$$

where

$$
L_{i}(q)=\frac{x q^{i} q^{(k+1-i)(k-i)}}{1-i x \prod_{j=i+1}^{k} q^{j}} .
$$

We have

$$
\lim _{q \rightarrow 1} \frac{d}{d q} L_{i}(q)=\frac{x \ell A_{i, k}(x, 1)-x \lim _{q \rightarrow 1} \frac{d}{d q} A_{i, k}(x, q)}{\left(A_{i, k}(x, 1)\right)^{2}} .
$$

Where $A_{i, k}(x, q)=1-i x \prod_{j=i+1}^{k} q^{j}$ and $\ell=(k+1-i)(k-i)+i$. By using the differentiation rules we get $\frac{d}{d q} A_{i, k}(x, q)=-i x \sum_{m=i+1}^{k} m q^{m-1} \prod_{\substack{j=i+1 \\ j \neq m}}^{k} q^{j}$. Therefore,

$$
\lim _{q \rightarrow 1} \frac{d}{d q} L_{i}(q)=\frac{x(2(i+(k+1-i)(k-i))(1-i x)+i(i+1+k)(k-i) x)}{2(1-i x)^{2}},
$$

which leads to

$$
\lim _{q \rightarrow 1}\left(\frac{\frac{d}{d q} L_{i}(q)}{L_{i}(q)}\right)=i+(k+1-i)(k-i)+\frac{i(i+1+k)(k-i) x}{2(1-i x)} .
$$

Hence, by substituting (5) in (3) we obtain

$$
\begin{aligned}
& \left.\frac{d}{d q} P_{k}(x, q)\right|_{q=1}=\frac{x^{k}}{(1-x) \ldots(1-k x)} \sum_{i=1}^{k}\left(i+(k+1-i)(k-i)+\frac{i(i+1+k)(k-i) x}{2(1-i x)}\right) \\
& =\frac{\left.x^{k}\left(\begin{array}{c}
k+1 \\
2
\end{array}\right)+2\left(\begin{array}{c}
k+1 \\
3
\end{array}\right)\right)}{(1-x) \ldots(1-k x)}+\frac{x^{k+1}}{(1-x) \ldots(1-k x)} \sum_{i=1}^{k} \frac{i(i+1+k)(k-i)}{2(1-i x)},
\end{aligned}
$$


as claimed.

Now we need to find $\left.\left[x^{n}\right] \frac{d}{d q} P_{k}(x, q)\right|_{q=1}$ to obtain the total number of swrec. We will study the exponential generating function instead of the ordinary generating function. Let $\widetilde{P}_{k}(x, u, q)$ be the exponential generating function for the number of partitions of $[n]$ with exactly $k$ blocks according to the statistic swrec, that is

$$
\widetilde{P}_{k}(x, u, q)=\sum_{n \geq k} \sum_{\pi \in P_{n, k}} \frac{x^{n} u^{k} q^{\operatorname{swrec}(\pi)}}{n !} .
$$

Theorem 3 The partial derivative of $\widetilde{P}_{k}(x, u, q)$ with respect to $q$ at $u=q=1$ is given by,

$$
\left.\frac{d}{d q} \widetilde{P}_{k}(x, u, q)\right|_{u=q=1}=e^{e^{x}-1}\left(\frac{3}{4} e^{3 x}+\frac{3}{2} e^{2 x}-\frac{7}{4} e^{x}-x e^{2 x}-\frac{3}{2} x e^{x}-\frac{1}{2}\right) .
$$

Proof In order to prove the above result we need the following proposition:

Proposition 4 The partial derivative $\left.\frac{d}{d q} P_{k}(x, q)\right|_{\substack{x=y^{-1} \\ q=1}}$ can be decomposed as

$$
\sum_{m=1}^{k}\left(\frac{a_{k, m}}{(y-m)^{2}}+\frac{b_{k, m}}{y-m}\right)
$$

where

$$
a_{k, m}=\frac{(-1)^{k-m} m(1+k+m)(k-m)}{2(m-1) !(k-m) !},
$$

and

$$
b_{k, m}=\frac{(-1)^{k-m}\left(k^{2}\left(\frac{m}{4}+1\right)+k\left(\frac{m^{2}}{2}+\frac{3 m}{4}+1\right)-\left(\frac{3 m^{2}}{2}+m\right)\right)}{(m-1) !(k-m) !} .
$$

Proof We rewrite (2) as

$$
\begin{aligned}
& \left.\frac{d}{d q} P_{k}(x, q)\right|_{q=1}=x^{k} \prod_{i=1}^{k}(1-i x)^{-1}\left(\left(\begin{array}{c}
k+1 \\
2
\end{array}\right)+2\left(\begin{array}{c}
k+1 \\
3
\end{array}\right)+\sum_{i=1}^{k} \frac{i(1+k+i)(k-i) x}{2(1-i x)}\right) \\
= & x^{k} \prod_{i=1}^{k}(1-i x)^{-1}\left(\frac{k(k+1)(2 k+1)}{6}+\sum_{i=1}^{k} \frac{i(1+k+i)(k-i) x}{2(1-i x)}\right) .
\end{aligned}
$$

By replacing $x^{-1}=y$ in the above equation we get

$$
\prod_{i=1}^{k}(y-i)^{-1}\left(\frac{k(k+1)(2 k+1)}{6}+\sum_{i=1}^{k} \frac{i(1+k+i)(k-i)}{2(y-i)}\right) .
$$


The above expression decomposed as

$$
\sum_{m=1}^{k}\left(\frac{a_{k, m}}{(y-m)^{2}}+\frac{b_{k, m}}{y-m}\right) .
$$

In order to find the coefficients $a_{k, m}$ and $b_{k, m}$, we need to consider the expansion of (8) at $y=m$, as follows:

$$
\begin{aligned}
& (y-m)^{-1} \prod_{\substack{i=1 \\
i \neq m}}^{k}(y-m+m-i)^{-1}\left(\frac{k(k+1)(2 k+1)}{6}+\frac{m(1+k+m)(k-m)}{2(y-m)}+\sum_{\substack{i=1 \\
i \neq m}}^{k} \frac{i(1+k+i)(k-i)}{2(y-i)}\right) \\
& =(y-m)^{-1} \prod_{\substack{i=1 \\
i \neq m}}^{k}\left((m-i)^{-1}\left(1+\frac{y-m}{m-i}\right)^{-1}\right) . \\
& \left(\frac{k(k+1)(2 k+1)}{6}+\frac{m(1+k+m)(k-m)}{2(y-m)}+\sum_{\substack{i=1 \\
i \neq m}}^{k} \frac{i(1+k+i)(k-i)}{2(y-i)}\right) .
\end{aligned}
$$

Using Taylor series to expand $\left(1+\frac{y-m}{m-i}\right)^{-1}$ and $\frac{i(1+k+i)(k-i)}{2(y-i)}$ at $y=m$ we get

$$
\begin{aligned}
& (y-m)^{-1} \frac{(-1)^{k-m}}{(m-1) !(k-m) !} \prod_{\substack{i=1 \\
i \neq m}}^{k}\left(1-\frac{y-m}{m-i}+O\left((y-m)^{2}\right)\right) \\
& \left(\frac{k(k+1)(2 k+1)}{6}+\frac{m(1+k+m)(k-m)}{2(y-m)}+\sum_{\substack{i=1 \\
i \neq m}}^{k} \frac{i(1+k+i)(k-i)}{2(m-i)}+O(y-m)\right),
\end{aligned}
$$

which is equivalent to

$$
\begin{aligned}
& (y-m)^{-1} \frac{(-1)^{k-m}}{(m-1) !(k-m) !}\left(1-\sum_{\substack{i=1 \\
i \neq m}}^{k} \frac{y-m}{m-i}+O\left((y-m)^{2}\right)\right) \\
& \cdot\left(\frac{k(k+1)(2 k+1)}{6}+\frac{m(1+k+m)(k-m)}{2(y-m)}+\sum_{\substack{i=1 \\
i \neq m}}^{k} \frac{i(1+k+i)(k-i)}{2(m-i)}+O(y-m)\right) .
\end{aligned}
$$

We need to simplify the product, and consider the coefficients of $(y-m)^{-1}$ and $(y-m)^{-2}$ as follows:

$$
\begin{aligned}
& (y-m)^{-1} \frac{(-1)^{k-m}}{(m-1) !(k-m) !} \\
& \cdot\left(\frac{k(k+1)(2 k+1)}{6}+\frac{m(1+k+m)(k-m)}{2(y-m)}+\sum_{\substack{i=1 \\
i \neq m}}^{k} \frac{i(1+k+i)(k-i)-m(1+k+m)(k-m)}{2(m-i)}+O(y-m)\right) .
\end{aligned}
$$


By using Maple we compute the term in the summation, which hints

$$
\begin{aligned}
& (y-m)^{-1} \frac{(-1)^{k-m}}{(m-1) !(k-m) !} \\
& \cdot\left(\frac{m(1+k+m)(k-m)}{2(y-m)}+\frac{k(k+1)(2 k+1)}{6}+\frac{1}{2} \sum_{\substack{i=1 \\
i \neq m}}^{k}\left(i^{2}+i+i m-k^{2}-k+m+m^{2}\right)+O(y-m)\right) \\
& =(y-m)^{-1} \frac{(-1)^{k-m}}{(m-1) !(k-m) !} \\
& \cdot\left(\frac{m(1+k+m)(k-m)}{2(y-m)}+k^{2}\left(\frac{m}{4}+1\right)+k\left(\frac{m^{2}}{2}+\frac{3 m}{4}+1\right)-\left(\frac{3 m^{2}}{2}+m\right)+O(y-m)\right) .
\end{aligned}
$$

Hence, by finding the coefficients of of $(y-m)^{-1}$ and $(y-m)^{-2}$ we complete the proof.

Now we return to the proof of the theorem. We use (7) for passing to exponential generating function, by substituting

$$
\frac{e^{m x}-1}{m}=\sum_{\ell \geq 0} \frac{m^{\ell} x^{\ell+1}}{(\ell+1) !}
$$

in

$$
\frac{1}{y-m}=\frac{x}{1-m x}=\sum_{\ell \geq 0} m^{\ell} x^{\ell+1}
$$

and $\frac{e^{m x}(m x-1)+1}{m^{2}}$ in $\frac{1}{(y-m)^{2}}$. Moreover, by summing over all $k$ we obtain the generating function

$$
\begin{aligned}
& \sum_{k \geq 1} u^{k} \sum_{m=1}^{k} \frac{(-1)^{k-m} m(1+k+m)(k-m)}{2(m-1) !(k-m) !} \cdot \frac{e^{m x}(m x-1)+1}{m^{2}} \\
& +\sum_{k \geq 1} u^{k} \sum_{m=1}^{k} \frac{(-1)^{k-m}\left(k^{2}\left(\frac{m}{4}+1\right)+k\left(\frac{m^{2}}{2}+\frac{3 m}{4}+1\right)-\left(\frac{3 m^{2}}{2}+m\right)\right)}{(m-1) !(k-m) !} \cdot \frac{e^{m x}-1}{m} .
\end{aligned}
$$

We need to change the order of the summation as follows:

$$
\begin{aligned}
& \sum_{m \geq 1} \frac{e^{m x}(m x-1)+1}{m !} \sum_{k \geq m} \frac{(-1)^{k-m}(1+k+m)(k-m) u^{k}}{2(k-m) !} \\
& +\sum_{m \geq 1} \frac{e^{m x}-1}{m !} \sum_{k \geq m} \frac{(-1)^{k-m}\left(k^{2}\left(\frac{m}{4}+1\right)+k\left(\frac{m^{2}}{2}+\frac{3 m}{4}+1\right)-\left(\frac{3 m^{2}}{2}+m\right)\right)}{(k-m) !} u^{k} .
\end{aligned}
$$

By substituting $\ell=k-m$ and rewriting the above result we obtain the following form:

$$
\begin{aligned}
& \sum_{m \geq 1} \frac{e^{m x}(m x-1)+1}{m !} \sum_{\ell \geq 0} \frac{(-1)^{\ell}(2 m+\ell+1) \ell u^{m+\ell}}{2 \ell !} \\
& +\sum_{m \geq 1} \frac{e^{m x}-1}{m !} \sum_{\ell \geq 0} \frac{(-1)^{\ell}\left((m+\ell)^{2}\left(\frac{m}{4}+1\right)+(m+\ell)\left(\frac{m^{2}}{2}+\frac{3 m}{4}+1\right)-\left(\frac{3 m^{2}}{2}+m\right)\right)}{\ell !} u^{m+\ell} .
\end{aligned}
$$


By evaluating the previous terms in $u=1$ we complete the proof.

Theorem 5 The total number of swrec taken over all set partitions of $[n]$, is given by

$$
\frac{3}{4}\left(B_{n+3}-B_{n+2}\right)-\left(n+\frac{7}{4}\right) B_{n+1}-\frac{1}{2}(n+1) B_{n} .
$$

Proof In order to find the total number of swrec, we need to find an explicit formula for the coefficient of $x^{n}$ in the generating function $\left.\frac{d}{d q} \widetilde{P}_{k}(x, u, q)\right|_{u=q=1}$. By Theorem 3

$$
\left.\frac{d}{d q} \widetilde{P}_{k}(x, u, q)\right|_{u=q=1}=e^{e^{x}-1}\left(\frac{3}{4} e^{3 x}+\frac{3}{2} e^{2 x}-\frac{7}{4} e^{x}-x e^{2 x}-\frac{3}{2} x e^{x}-\frac{1}{2}\right) .
$$

By differentiating the well known generating function $e^{e^{x}-1}=\sum_{n \geq 0} B_{n} \frac{x^{n}}{n !}$ three times we obtain

$$
\begin{gathered}
e^{x} e^{e^{x}-1}=\sum_{n \geq 0} B_{n+1} \frac{x^{n}}{n !}, \\
e^{2 x} e^{e^{x}-1}=\sum_{n \geq 0} B_{n+2} \frac{x^{n}}{n !}-\sum_{n \geq 0} B_{n+1} \frac{x^{n}}{n !}
\end{gathered}
$$

and

$$
e^{3 x} e^{e^{x}-1}=\sum_{n \geq 0} B_{n+3} \frac{x^{n}}{n !}-3 \sum_{n \geq 0} B_{n+2} \frac{x^{n}}{n !}+2 \sum_{n \geq 0} B_{n+1} \frac{x^{n}}{n !} .
$$

From the above equations, we can derive that

$$
x e^{x} e^{e^{x}-1}=\sum_{n \geq 0} n B_{n} \frac{x^{n}}{n !}
$$

and

$$
x e^{2 x} e^{e^{x}-1}=\sum_{n \geq 0} n B_{n+1} \frac{x^{n}}{n !}-\sum_{n \geq 0} n B_{n} \frac{x^{n}}{n !} .
$$

Using all these facts together leads to

$$
\left.\frac{d}{d q} \widetilde{P}_{k}(x, u, q)\right|_{u=q=1}=\sum_{n \geq 0}\left(\frac{3}{4} B_{n+3}-\frac{3}{4} B_{n+2}-\left(n+\frac{7}{4}\right) B_{n+1}-\frac{1}{2}(n+1) B_{n}\right) \frac{x^{n}}{n !} .
$$

Hence the total number of swrec is given by

$$
\frac{3}{4}\left(B_{n+3}-B_{n+2}\right)-\left(n+\frac{7}{4}\right) B_{n+1}-\frac{1}{2}(n+1) B_{n} .
$$

In order to obtain asymptotic estimate for the moment as well as limiting distribution, we need the fact

$$
B_{n+h}=B_{n} \frac{(n+h) !}{n ! r^{h}}\left(1+O\left(\frac{\log n}{n}\right)\right)
$$

uniformly for $h=O(\log n)$, where $r$ is the positive root of $r e^{r}=n+1$. For more details about the asymptotic expansion of Bell numbers see [1. Therefore, Theorem 5 gives the following corollary. 
Corollary 6 Asymptotically, the total number of swrec taken over all set partitions of $[n]$, is given by

$$
B_{n} \frac{n^{3}}{r^{3}}\left(1+\frac{r}{n}\right)\left(1+O\left(\frac{\log n}{n}\right)\right)
$$

Acknowledgement. The research of the author was supported by the Ministry of Science and Technology, Israel.

\section{References}

[1] E.R. Canfield, Engel's inequality for Bell numbers, J. Combin. Theory Ser. A 72 (1995), no.1, $184-187$.

[2] N. Glick, Breaking records and breaking boards, Amer. Math. Monthly 85 (1978), no.1, 2-26.

[3] A. Knopfmacher and T. Mansour, Record statistics in a random composition, Discrete Appl. Math. 160 (2012), no.4-5, 593-603.

[4] A. Knopfmacher, T. Mansour and S. Wagner, Records in set partitions, Electron. J. Combin. 17 (2010), no.1, Paper 109, 14 pp.

[5] I. Kortchemski, Asymptotic behavior of permutation records, J. Combin. Theory Ser. A 116 (2009), no.6, 1154-1166.

[6] T. Mansour, Combinatorics of Set Partitions, CRC Press, Boca Raton, FL, 2013.

[7] A. Rényi, Théorie des éléments saillants d'une suite d'observations, Ann. Fac. Sci. Univ. Clermont-Ferrand 8 (1962) 7-13.

[8] R. P. Stanley, Enumerative Combinatorics, Vol. 1, Cambridge University Press, Cambridge, UK, 1996. 